\title{
Magnetic track array for efficient bead capture in microchannels
}

\author{
Mélanie Abonnenc • Anne-Laure Gassner • \\ Jacques Morandini • Jacques Josserand • \\ Hubert H. Girault
}

Received: 30 April 2009/Revised: 20 July 2009 /Accepted: 23 July 2009/Published online: 16 August 2009

(C) Springer-Verlag 2009

\begin{abstract}
Magnetism-based microsystems, as those dedicated to immunoaffinity separations or (bio)chemical reactions, take benefit of the large surface area-to-volume ratio provided by the immobilized magnetic beads, thus increasing the sensitivity of the analysis. As the sensitivity is directly linked to the efficiency of the magnetic bead capture, this paper presents a simple method to enhance the capture in a microchannel. Considering a microchannel surrounded by two rectangular permanent magnets of different length $\left(L_{\mathrm{m}}=2,5,10 \mathrm{~mm}\right)$ placed in attraction, it is shown that the amount of trapped beads is limited by the magnetic forces mainly located at the magnet edges. To overcome this limitation, a polyethylene terephthalate (PET) microchip with an integrated magnetic track array has been prototyped by laser photo-ablation. The magnetic force is therefore distributed all along the magnet length. It results in a multi-plug bead capture, observed by microscope imaging, with a magnetic force value locally enhanced. The relative amount of beads, and so the specific binding surface for further immunoassays, presents a significant increase of $300 \%$ for the largest magnets. The influence of the track geometry and relative permeability on
\end{abstract}

Electronic supplementary material The online version of this article (doi:10.1007/s00216-009-3006-3) contains supplementary material, which is available to authorized users.

M. Abonnenc · A.-L. Gassner · J. Josserand $\cdot$ H. H. Girault $(\bowtie)$

Laboratoire d'Electrochimie Physique et Analytique,

Ecole Polytechnique Fédérale de Lausanne (EPFL)-SB-ISIC-

LEPA, Station 6,

1015 Lausanne, Switzerland

e-mail: hubert.girault@epfl.ch

J. Morandini

Astek Rhône-Alpes,

1 place du Verseau,

38130 Echirolles, France the magnetic force was studied by numerical simulations, for the microchip operating with 2-mm-long magnets.

Keywords Microfluidics · Magnetism · Permanent magnet . Magnetic bead · Ink · Magnetic track array · Photo-ablation . Polymer $\cdot$ Numerical simulation

\section{Introduction}

Target analytes in biological samples are often present in low concentrations relative to the surrounding matrix components, necessitating effective separation techniques. Nowadays, immunoaffinity separation is a widespread tool in clinical testing, diagnostic, and bio-chemical sample analysis. Whereas enzyme-linked immunosorbent assay (ELISA) tests require at best few minutes for easy-to-use formats and hours for classical microtiter well tests, immunoassays in microfluidic format present the advantages of reduced reagent consumption and fast time of analysis [1]. Proteins may be either attached directly to the walls of a capillary column or microchip channel, or to solid-phase supports and packed into columns or channels. Solid support may consist of particles or beads made from plastic, silica or glass, and magnetic materials. Protein adsorption in polymer microchannels [2] and its dynamic under stopped flow and continuous flow have been previously investigated in our lab [3, 4]. Alternatively, magnetic beads were also used as solid-phase support to develop magnetic bead-based immunoassay both in microchip for biosensing and in capillary for immunoaffinity capillary electrophoresis (IA-CE) [5-7]. As the sensitivity of the analysis is highly dependent on the magnetic beadtrapping efficiency, we propose here a novel design of photo-ablated polymer microchip integrating a magnetic 
track array to enhance the magnetic bead capture in a microchannel.

Magnetic beads are usually superparamagnetic particles that exhibit a high magnetization in presence of a magnetic field, allowing an easy manipulation of the particles. Once the external magnetic field removed, they present no residual magnetism and can be freely re-suspended [8]. A wide range of bioreactive molecules can be adsorbed or coupled to the bead-surface, which provides a high-binding surface area. Yet, they have proven to be efficient tools in medical applications such as drug targeting $[9,10]$ and bioseparations including protein/peptide isolation, bioassays, or cell sorting [11, 12]. Typically, isolation of bio-molecules, such as peptides and proteins, is usually performed using a variety of chromatography, electrophoretic, ultrafiltration, precipitation techniques. The use of magnetic particles for this purpose can simplify considerably the purification steps, limiting as well the volume of eluting or precipitation buffers [13]. Whereas the magnetic beads are nowadays commonly used at laboratory-scale (i.e., in tube), the development of methodologies in microfluidic format has recently emerged decreasing sample/reagent consumption, cost and time consumption [14-17]. Such magnetism-based devices have found great applications in biology, as for cell manipulation [18-23], (bio)chemical reaction as proteolysis [24, 25], bioassay [26-28], DNA or RNA hybridization [29, 30]. Magnetic beads appeared as well in microsystems to design mixers, valves, or switches [31-37].

The microfabrication complexity of such magnetismbased devices is highly dependent to whether the magnetic field actuation is integrated or not to the device. Active magnetic microsystems rely on the use of on-chip microelectromagnets capable of a local addressability [15]. However, the complex microfabrication processes and the limited field strength $(0-100 \mathrm{mT})$ are the weak points of these devices. In contrast, passive magnetic microsystems use external macro-sized permanent magnets or electromagnets $[25,27,38]$. The process is simplified and larger magnetic fields $(>0.5 \mathrm{~T})$ and forces can be reached. In a recent study, the distribution of magnetic field and force in a microchannel surrounded by permanent magnets was simulated [39]. It has been shown that the magnetic field and force is restricted to a specific location defined by the magnet geometry and pole orientation (attraction, repulsion, single magnet). In order to control the magnetic field addressing towards specific locations, magnetic elements can be integrated to the structure, both in active and passive microsystems [40, 41]. Smistrup et al. introduced an onchip magnetic bead microarray in which an external magnetic field $(21 \mathrm{mT})$ magnetizes soft magnetic elements placed along the microchannel [29]. In combination with hydrodynamic focusing, the beads from different incoming streams are captured at the sidewalls and functionalized with two types of magnetic beads carrying different probes to assess DNA hybridization. Alternatively to their work in which the magnetic field value limits the bead trapping at the sidewalls, the present study uses a higher field to magnetize the elements leading to full multi-plugs of beads. The fabrication process is also simpler and based on polymer laser ablation. Indeed, in contrast with intensive clean-room and multi-steps fabrication processes as siliconbased techniques, fast prototyping such as soft lithography [42] and polymer laser ablation [43, 44] are quite simple, cheap, and in most cases, do not require a heavy cleanroom environment. Several groups have used soft lithography to design magnetism-based devices [42, 45]. Viovy et al. introduced a PDMS microchip, based on soft lithography and fast prototyping, with embedded permanent magnets oriented in repulsion from $20^{\circ}$ with respect to the microchannel axis [25]. The chip was demonstrated to be an efficient reactor to perform protein digestion thanks to the high surface area-to-volume ratio provided by the beads that are organized in columns parallel to the flow, thus limiting the flow resistance.

Taking advantage of our lab experience in polymer laser photo-ablation [46-48], a microchip, including a magnetic track array, was designed in order to get an efficient bead capture along the microchannel for further use in immunoaffinity separation (Fig. 1). The actuation is made by two face-to-face permanent magnets placed in attraction and fixed on both microchannel sides to magnetize the tracks filled with a home-prepared magnetic ink. The magnetic field is therefore addressed through these tracks, symmetrically located as an array perpendicular to the microchannel, resulting in a multi-plug bead capture. The study includes microscope imaging showing the multi-plug bead organization in microchips including a magnetic track array. By comparison with a microchip without array, the gain of using multi-plugs vs. a single plug of beads is evaluated, when magnets of 2-, 5-, and 10-mm long are used. Numerical simulations of the inter-track gap, channel-track gap, and ink permeability provide an orientation to improve this gain.

\section{Theory}

Magnetic particle transport in a microfluidic system

The present investigation describes the physical phenomena occurring in a microfluidic system constituted of a microchannel surrounded by two face-to-face permanent magnets in attraction (Fig. 1). The superparamagnetic beads in solution are infused under pressure-driven flow from the microchannel left side.

The main forces experienced by the particles are the magnetic force, $\boldsymbol{F}_{\mathbf{m a g}}$ and the hydrodynamic drag force, 


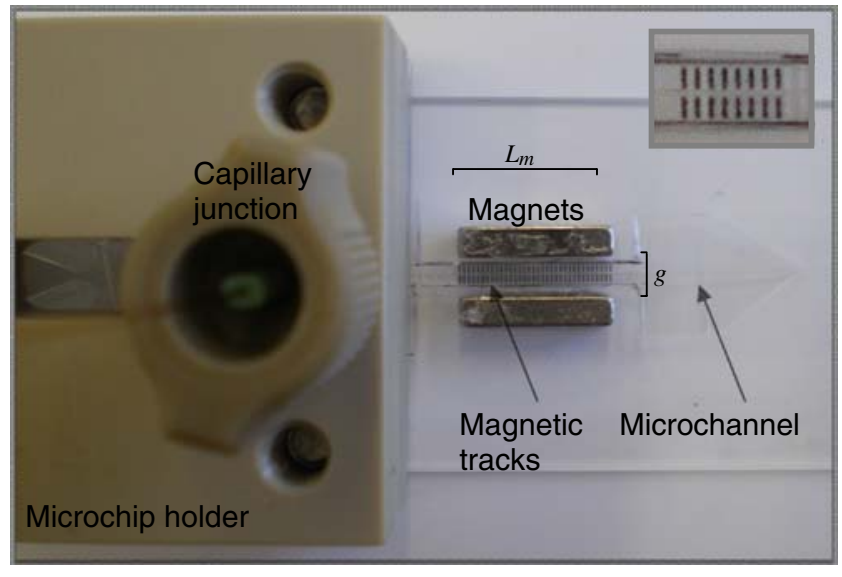

Fig. 1 Top-view of the microchip with magnetic tracks. The microchannel (100 $\mu \mathrm{m}$ wide, $50 \mu \mathrm{m}$ deep, $4 \mathrm{~cm}$ long) is positioned in a holder with a capillary connection fittings. The magnetic track array is composed of 34 tracks spaced by $200 \mu \mathrm{m}$ and is surrounded by two permanent magnets of $10 \mathrm{~mm}$ long fixed in attraction on a glass slide. $L_{\mathrm{m}}$ is the magnet length and $g$ the inter-magnet gap. The inset picture shows eight magnetic tracks spaced by $200 \mu \mathrm{m}$ designed for $2 \mathrm{~mm}$ long magnets, which is the study case in the numerical simulations

$\boldsymbol{F}_{\text {drag. }}$. The gravitational force, particle/fluid interactions and inter-particle effects are neglected.

In a material submitted to a magnetic field $\boldsymbol{H}$ generated by a magnetic source, the local flux density (or induction) $\boldsymbol{B}$ is the sum of the induction $\mu_{0} \boldsymbol{H}$ given by the source in the vacuum and of the local induction $\mu_{0} \boldsymbol{M}$ due to the magnetization $\boldsymbol{M}$ of the studied medium $(\boldsymbol{M}=\chi \boldsymbol{H}$ in the linear magnetization zone of the material):

$\boldsymbol{B}=\mu_{0}(\boldsymbol{H}+\boldsymbol{M})=\mu_{0}(1+\chi) \boldsymbol{H}=\mu_{0} \mu_{\mathrm{r}} \boldsymbol{H}=\mu \boldsymbol{H}$

where $\mu_{0}$ and $\mu$ are the respective vacuum and bead absolute permeability, $\mu_{r}$ the relative bead permeability and $\chi$ the magnetic susceptibility of the bead material. For a superparamagnetic bead of volume $V$ immersed in a solution, the magnetic moment is given by $\boldsymbol{m}_{\text {bead }}=V \boldsymbol{M}=$ $V \Delta \chi \boldsymbol{H}$ where $\Delta \chi=\chi-\chi_{\text {water }}$ is the relative susceptibility of the bead compared to the one of the solution. The magnetic force applied on these particles can be expressed as the derivative of the magnetic potential energy:

$\boldsymbol{F}_{\text {mag }}=\nabla\left(\boldsymbol{m}_{\text {bead }} \cdot \boldsymbol{B}\right) \approx\left(\boldsymbol{m}_{\text {bead }} \cdot \nabla\right) \boldsymbol{B}$

which results in Eq. 3 by taking into account Eq. 1 and the moment expression under the saturation conditions of the beads $\left(\boldsymbol{m}_{\text {bead }} \ll \boldsymbol{m}_{\text {sat }}\right)$ :

$\boldsymbol{F}_{\text {mag }}=\frac{V \Delta \chi}{\mu_{0}}(\boldsymbol{B} \cdot \nabla) \boldsymbol{B}$

In addition to the magnetic force, the particles experience a hydrodynamic drag force, defined by the Stokes' equation,

$\boldsymbol{F}_{\text {drag }}=6 \pi \eta R v$ with the assumption that the magnetic beads have the approximate shape of a sphere of radius $R, \eta$ is the fluid viscosity and $v$ the velocity of the bead compared to the surrounding fluid velocity.

\section{Numerical simulations}

Numerical simulations of the magnetic field and force distribution, based on the finite elements (FE) method, were carried out in a 2D geometry, in magnetostatic conditions. The simulations were performed without any flow in the microchannel. The FE formulation was implemented in the commercial software Flux-Expert ${ }^{\mathrm{TM}}$ (Astek, Rhône-Alpes, Grenoble, France) on a Mac Pro with Ubuntu Linux 7.10 operating system.

\section{Numerical model and assumptions}

As the different study domains are nonconductive (without any free electrical current), the first Maxwell equation writes $\nabla \wedge \boldsymbol{H}=0$ and consequently the magnetic field can be derived from the magnetic scalar potential $\phi(\boldsymbol{H}=-\nabla \phi)$. From the magnetic induction conservation and Eq. 1 in which the permanent induction $\boldsymbol{B}_{\text {mag }}$ imposed by the magnet is added, we have:

$$
\nabla \cdot \boldsymbol{B}=\nabla \cdot\left(-\mu \nabla \phi+\boldsymbol{B}_{m a g}\right)=0
$$

which is solved by using a Galerkin formulation (Supporting information S1). In any point of the domain, the flux density vector $\boldsymbol{B}$ is deduced from the $\phi$ value.

The following assumptions are made: (a) magnetostatic conditions $(\partial \boldsymbol{B} / \partial t=0)$, (b) 2D Cartesian form: the third dimension of the system (i.e., the magnet channel and track thickness), is supposed to be at least ten times larger than its height (i.e., magnet gap), so it can be neglected), (c) no magnetic elements around the magnets, (d) homogeneous media ( $\mu$ uniform in each of the different domains), (e) stopped-flow conditions (i.e. static bead solution in the microchannel), (f) the particle-fluid or inter-particle interaction are not considered. $(\mathrm{g})$ The effect of the beads on the solution permeability $\left(\mu_{\text {solution }}\right)$ is neglected. (h) The magnetic beads are assumed to be ideal $\left(\boldsymbol{m}_{\mathrm{sat}}>>\boldsymbol{B}_{\max }\right)$ as there is no quantitative comparison with experimental results.

This numerical model has been previously validated showing a good correlation with published results [39, 49].

Geometry and numerical parameters

The reference geometry considers two face-to-face permanent macro-magnets $\left(L_{\mathrm{m}}=2 \mathrm{~mm}\right)$ placed in attraction with 
an inter-magnet gap $g=2 \mathrm{~mm}$, as illustrated in Fig. 1. Consequently, the magnet length over inter-magnet gap ratio $L_{\mathrm{m}} / g=1$. The microchip presents a thinner width of $1.3 \mathrm{~mm}$ in the magnet region and includes at its center a 100- $\mu \mathrm{m}$-wide microchannel. The following parameters are applied on the studied geometry: relative permeability of the materials $\mu_{r}=1$ in the entire domain, except in the magnetic tracks where $\mu_{r}=4$. Imposed flux density in the magnets: $\mathbf{B}_{\text {mag }}= \pm 1.3 \mathrm{~T}$. The beads parameters are fixed at unity: $\Delta \chi=1, \quad V=1.4 \cdot 10^{-20} \mathrm{~m}^{3}$ (for a bead diameter of $0.3 \mu \mathrm{m})$. An "air box" is meshed around the magnets to give enough space for the magnetic field rotation from one pole to the other. The size of the mesh elements and of the "air box" has been systematically calibrated.

The numerical investigations concerning the microchip with magnetic tracks were restricted to the design with 2-mm-long magnets as the number of tracks to mesh would be too high for larger magnets.

\section{Experimental}

\section{Chemicals}

Three different magnets were used for this study: a rectangular $\mathrm{NdFeB} 2 \times 2 \times 8 \mathrm{~mm}^{3}$ magnet (magnetic remanence of $1.3 \mathrm{~T}$ at the pole and a polarization in the longest dimension, from Chen Yang, Finsing, Germany), two rectangular $5 \times 2.5 \times 2 \mathrm{~mm}^{3}$ and $10 \times 3 \times 2 \mathrm{~mm}^{3}$ magnets (magnetic remanence of $1.3 \mathrm{~T}$ at the pole, with a polarization in the smallest dimension, from Supermagnet, Switzerland). Fused-silica capillaries (100/375 $\mu \mathrm{m}$ i.d./o.d.) were obtained from BGB Analytik AG (Böckten, Switzerland). Protein A-coated superparamagnetic beads of uniform size (mean diameter of $300 \mathrm{~nm}$, binding capacity $100 \mu \mathrm{g} / \mathrm{mL}$ ) were purchased from Ademtech (Pessac, France). The bead suspensions were sonicated and diluted ten times in water produced by an alpha Q Millipore System (Zug, Switzerland).

Magnetic ink preparation and characterization

The home-prepared magnetic ink is made of $1 \mathrm{~g}$ of carbon ink (Electrador, Electra Polymer \& Chemicals Ltd., UK) mixed with $200 \mathrm{mg}$ of $10 \mu \mathrm{m}$ iron particles (Merck, Darmstadt, Germany). The density and magnetic permeability of the ink were determined. The density of $4.9679 \mathrm{~g} / \mathrm{cm}^{3}$ was measured with a helium pycnometer on the cured ink. The mass magnetization of the ink was measured with a SQUID as a function of the field at $300 \mathrm{~K}$. (Supporting information S2) The resulting value of dimensionless magnetic permeability was 4.07 . This value was used in the model.

\section{Device microfabrication}

The microfabrication process is based on polymer photoablation and is illustrated in Fig. 2.

Step 1. The main microchannel (100 $\mu \mathrm{m}$ wide, $50 \mu \mathrm{m}$ deep and $4 \mathrm{~cm}$ long) is drilled on the topside of a polyethylene-terephthalate sheet of $100 \mu \mathrm{m}$ thickness (Melinex sheet from Dupont, Wilmington, DE, USA) by photo-ablation with an ArF excimer laser (193 nm, Lambda Physik, Göttingen, Germany). Spaced by $650 \mu \mathrm{m}$ from the microchannel $x$ axis, a parallel channel $(50 \mu \mathrm{m}$ wide, $100 \mu \mathrm{m}$ deep and $1 \mathrm{~cm}$ long) is drilled to further facilitate the microchip lateral sides cutting. Then, rectangular tracks $\left(100 \times 500 \times 100 \mathrm{\mu m}^{3}\right)$ perpendicular to the main microchannel are drilled by static laser shoots. The reference design is defined by a channel-track gap of $10 \mu \mathrm{m}$ and an intertrack gap of $200 \mu \mathrm{m}$. Only the photo-ablation step is made in a clean-room.

Step 2. The chip is cleaned with $\mathrm{MeOH}$ and the lateral sides of the chip are removed to allow the later permanent magnet positioning close to the magnetic tracks. The chip is laminated and cured $1 \mathrm{~h}$ at $80{ }^{\circ} \mathrm{C}$.

Step 3. On the PET chip backside, the microchannel inlets are protected and the tracks are filled with the home-prepared magnetic ink.

Step 4. The backside of the chip is laminated and the chip is cured $30 \mathrm{~min}$ at $80{ }^{\circ} \mathrm{C}$.

The entire process is achieved in a single day with around ten microchips per batch.

Microfluidic system operation and cleaning

The permanent magnets are fixed on a microscope slide in attraction with an inter-magnet gap of $2 \mathrm{~mm}$. The microchip is then positioned in the inter-magnet gap, as illustrated in Fig. 2.

A PACE MDQ system (Beckman-Coulter, Nyon, Switzerland) embedding an autosampler and UV detector is used for the sample delivery, in pressure mode. The influence of the capillary connections to the microchip and the magnetic beads on the pressure drop was evaluated. (Supporting information S3)

The beads are captured at a flow rate of $100 \mathrm{~nL} / \mathrm{min}$, optimum to avoid beads sedimentation and unspecific binding during the filling. The time of injection was varied according to the experiments. For cleaning, the beads are flushed out after magnet removing. The chip is washed with acidic solutions or solvents, and if necessary immersed in ultra-sonic bath. 
Fig. 2 Microchip fabrication process

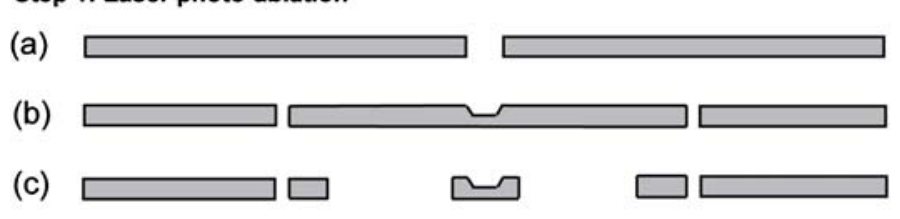

Step 2: Lamination and cutting $+1 \mathrm{~h} @ 80^{\circ} \mathrm{C}$

(a)

(b)

(c)

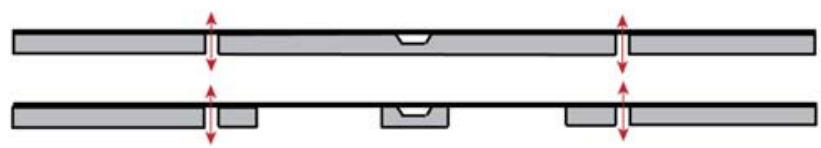

Step 3: Magnetic ink filling

(a)

(b)

(c)

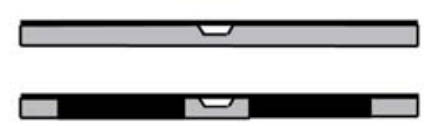

Step 4: Inlet protection and lamination + 30min @80 $0^{\circ} \mathrm{C}$

(a)

(b)

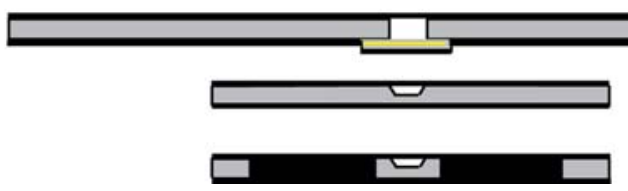

Imaging of magnetic bead capture/release

The magnetic beads were observed with a microscope Axiovert 200 (Carl Zeiss, Gottingen, Germany) and a CCD-IRIS camera (Sony, Tokyo, Japan). Images were processed with Igor 6.0 software (WaveMetrics, Portland, OR, USA).

\section{Results and discussion}

Forces and magnet length

Figure 3 a illustrates the variation of the magnetic force $(x$ component), along a microchannel surrounded by two permanent magnets of length $L_{\mathrm{m}}=2,5$, or $10 \mathrm{~mm}$. The magnets, in attractive configuration, present a symmetry axis at $x=0 . \boldsymbol{F}_{\text {mag }}$ is positive at the magnet left-edge and negative at the right-edge. For small magnets $\left(L_{\mathrm{m}}=2 \mathrm{~mm}\right)$, the zero- $\boldsymbol{F}_{\text {mag }}$ is restricted to a point located at the symmetry axis. When $L_{\mathrm{m}}>2 \mathrm{~mm}$, the zero- $\boldsymbol{F}_{\text {mag }}$ region becomes larger.

The main forces experienced by a magnetic bead in such a microfluidic system with large magnets $\left(L_{\mathrm{m}}=10 \mathrm{~mm}\right)$ are summarized in Fig. 3b. The microchannel region between the magnets can be subdivided by three where: (1) $\boldsymbol{F}_{\text {mag }}$ and $\boldsymbol{F}_{\text {drag }}$ are in the same direction resulting in the acceleration of the beads; (2) $\boldsymbol{F}_{\text {mag }}=0$, leading to the beads slow down; (3) $\boldsymbol{F}_{\text {mag }}$ is opposite to $\boldsymbol{F}_{\text {drag }}$. Consequently, if $\boldsymbol{F}_{\text {mag }}>\boldsymbol{F}_{\text {drag }}$, the beads will be trapped in this area. In stopped-flow conditions, the beads move back to the middle of the magnets, as the negative $\boldsymbol{F}_{\text {mag }} x$ component repels them.

The bead capture, in a microchannel surrounded by two $10 \mathrm{~mm}$-long magnets in attraction, was followed by microscope imaging. The next conditions were fixed for the entire study: (a) the flow velocity was kept constant for the bead delivery and only the bead injection time was changed according to the experiment; (b) the distance between the two magnets was kept constant $(g=2 \mathrm{~mm})$ to allow the microscopic imaging of the beads. In agreement with the force distribution, the beads are trapped from the magnet end-edge, as illustrated in Fig. 3c.

As the major magnet length is not optimally exploited in such conditions, our approach was to integrate a magnetic track array in order to distribute the magnetic field all along the microchannel.

Magnetic track integration

The microchip design and fabrication process are displayed in Figs. 1 and 2, and detailed in the "Experimental" section. Briefly, the microchip is constituted of a main microchannel 
Fig. 3 a Variation of the magnetic force ( $x$ component) in function of the magnet length $\left(L_{\mathrm{m}}=2,5\right.$ and $\left.10 \mathrm{~mm}\right)$.

b Scheme summarizing the main forces, $\boldsymbol{F}_{\mathbf{m a g}}$ and $\boldsymbol{F}_{\text {drag }}$, experienced by the magnetic beads, with large magnets $\left(L_{\mathrm{m}}>2 \mathrm{~mm}\right)$. c Microscope picture of the magnetic bead in a microchannel surrounded by two magnets of $L_{\mathrm{m}}=10 \mathrm{~mm}$ a

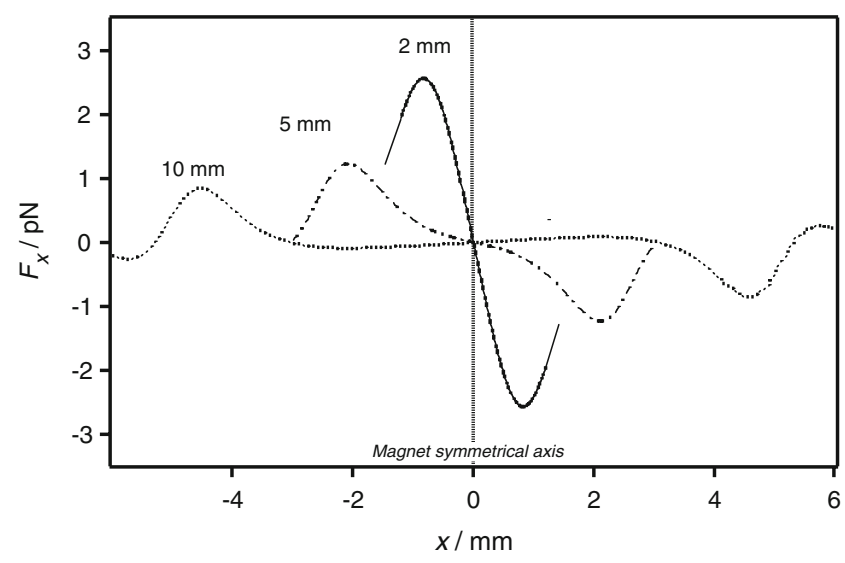

b

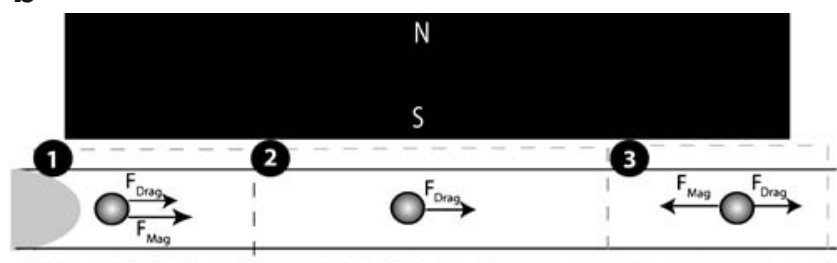

N

S

c

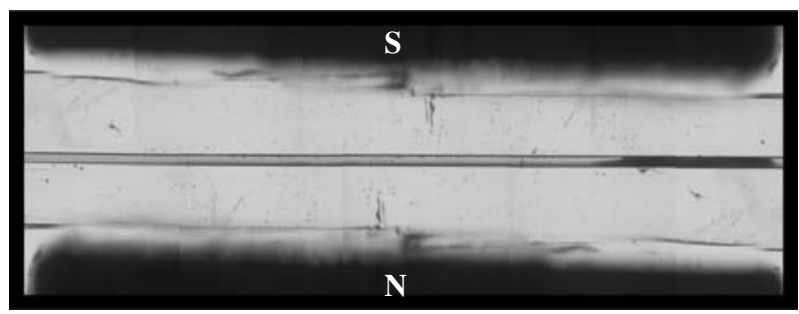

magnitude along the microchannel $(y=0)$ presents a maximum at the magnet vertical symmetry axis $(x=0)$. Along the microchannel $(y=0)$, the magnitude decreases near the magnet edges due to the enlarging of the field lines at the magnet extremities. The field line curvatures are at the origin of the $\boldsymbol{F}_{\text {mag }}$ force maxima in these regions (due to the $\mathbf{B} \nabla \mathbf{B}$ term in Eq. 3) [8, 39].

In the presence of magnetic tracks (Fig. 4b), the magnetic field lines are focused through the tracks and amplified because of the field line concentration. This configuration is analogous to one with successive micromagnets positioned along a microchannel where each track is comparable to a single magnet. However, when the external magnetic field is generated by permanent magnets, the magnetic field is not equally distributed between the tracks (as shown by the force in Fig. 5c). Then, the inner tracks experience a higher magnetic field than the outer ones. However, compared to on-chip micro- 


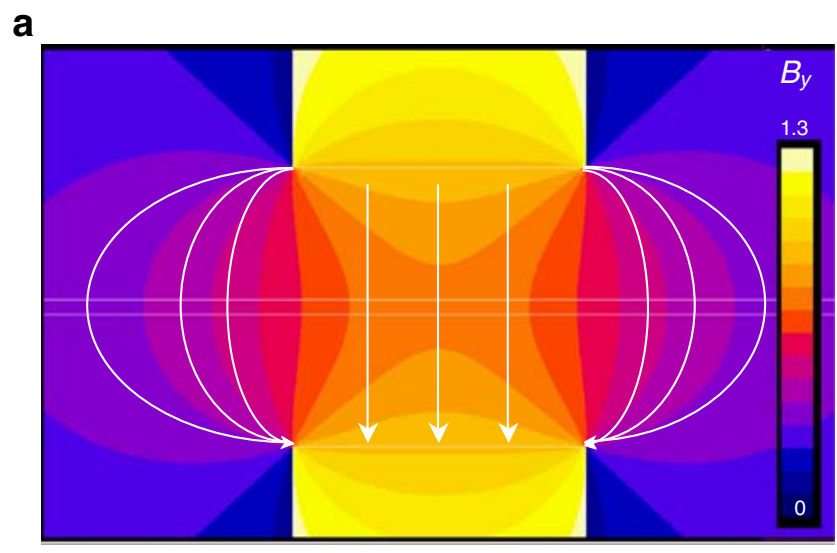

b

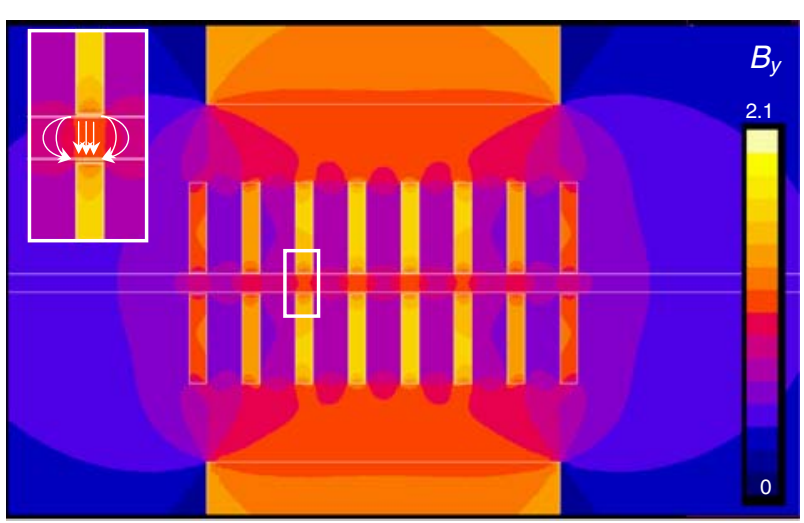

Fig. 4 Isovalues of the magnetic field distribution ( $y$ component). Microchips a without and $\mathbf{b}$ with eight magnetic tracks are simulated with 2-mm-long permanent magnets. The inter-magnet gap is $2 \mathrm{~mm}$ and channel width $100 \mu \mathrm{m}$. The white lines underline the curvatures of the magnetic field lines

electromagnets, the use of macro-sized permanent magnets combined with magnetic tracks enables to reach higher magnetic field values $(>0.5 \mathrm{~T})$.

Magnetic force mapping and resulting magnetic bead capture

Figure 5 shows the magnetic force distribution and the bead capture along the microchannel. Without tracks (Fig. 5a-b), the negative value of $\boldsymbol{F}_{\text {mag }}$ at the magnet end-edge counterbalances the $\boldsymbol{F}_{\text {drag }}$ magnitude and enables the magnetic bead trapping (assuming that $\boldsymbol{F}_{\text {mag }}>\boldsymbol{F}_{\text {drag }}$ ). For low flow rates (low value of $\boldsymbol{F}_{\text {drag }}$ ) the starting position of the plug (S) is located approximately at the middle of the magnet, as qualitatively confirmed on Fig. 5b. The beads are organized in a single plug growing with time until reaching the point where $\boldsymbol{F}_{\mathbf{m a g}}$ is not sufficient to retain them. It also confirms the partial bead release outside the end-side (E) of the plug. For the flow velocity value applied here, the region where $\boldsymbol{F}_{\text {mag }}>\boldsymbol{F}_{\text {drag }}$ (defined as the plug length $L_{\mathrm{P}}$ in dynamic conditions) represents $80 \%$ of the magnet length $L_{\mathrm{m}}$ (Fig. 5a-b).
Using the magnetic track configuration (Fig. $5 \mathrm{c}-\mathrm{d}$ ), the magnetic field distribution generates a pattern of successive positive-negative peaks of $\boldsymbol{F}_{\text {mag }}$ along the microchannel. The negative peaks located at each track allow to locally trap the beads, with a $\boldsymbol{F}_{\text {mag }} x$ component amplitude ten times higher. The first plug is growing from the first magnetic track. When saturation occurs, the beads move to fill the next plug, and so on.

\section{Design considerations}

The influence of the channel-track gap, inter-track gap and magnetic ink permeability values on the magnetic force is presented in Fig. 6. The results are evaluated as a gain, calculated from the ratio of magnetic forces between the chip embedding tracks and not. The position of the permanent magnets with respect to the microchannel remains unchanged. The force is considered at the maximal value at the center of the microchannel.

First, the channel-track vertical gap was increased from 1 to $25 \mu \mathrm{m}$. The gain, in terms of magnetic force, was evaluated for the $x$ - and $y$ components of the force, as illustrated in Fig. 6a and b, respectively. The first observation from both figures is that closer the magnetic tracks from the microchannel, larger are the local magnetic force value. In the presence of the magnetic tracks, the minimal $F_{x}$ gain is 10 for a gap of $25 \mu \mathrm{m}$ and can reach 19 for a gap of $1 \mu \mathrm{m}$ (Fig. 6a). Because of the fixed permanent magnets position, the addition of tracks has a major effect on the $y$ component of the force with values reaching three orders of magnitude. The $F_{y}$ gain is of $25 \times 10^{3}$ for a gap of $1 \mu \mathrm{m}$ and rapidly decreases to $1 \times 10^{3}$ for a $25-\mu \mathrm{m}$ gap (Fig. 6b). To illustrate the effect of the track vertical position on the $y$ component of the force, a chip was designed with an asymmetrical channel-track distance, as shown on the microscope image in Fig. 6b. When one of the magnetic tracks is closer to the microchannel, the beads start to form a cluster towards its sidewall resulting in an asymmetrical plug. From a microfabrication point of view, the reference design value was fixed at $10 \mu \mathrm{m}$ to be sure that the lamination layer resists to the pressure-driven flow.

The second studied parameter was the inter-track gap value, which was varied from 50 to $300 \mu \mathrm{m}$ (Fig. 6c). The number of tracks was adjusted to fit the magnet length. The tracks were positioned symmetrically from the magnet centre. Therefore, when the gap is small, the number of tracks in the area between the two permanent magnets becomes higher. When the gap is larger, the $F_{x}$ gain also increases from five to 17 and approaches a plateau for a gap value higher than $300 \mu \mathrm{m}$. For a gap of $50 \mu \mathrm{m}$, the position of the tracks are so close that they can be assimilated to a global magnet without changing significantly the magnetic field lines distribution. On the other hand, with a larger 
a

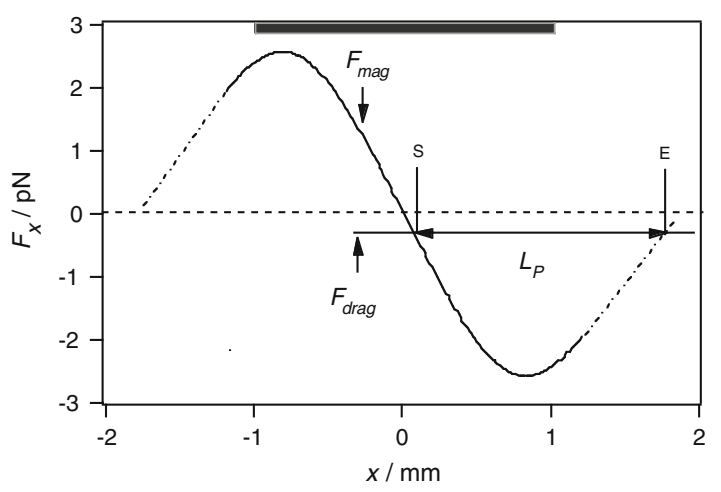

C

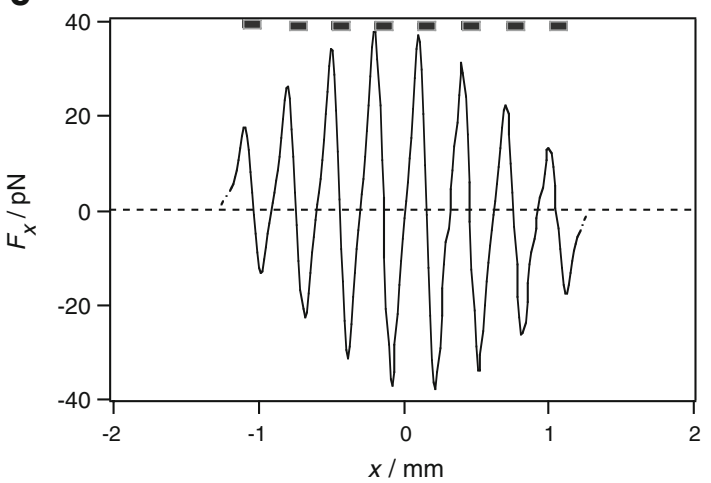

b

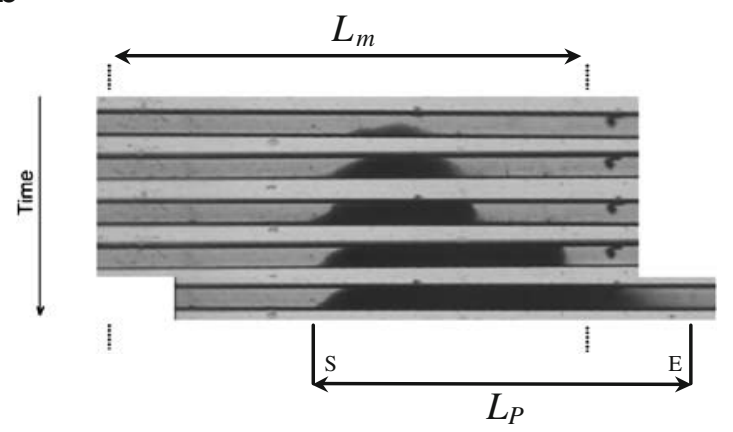

d

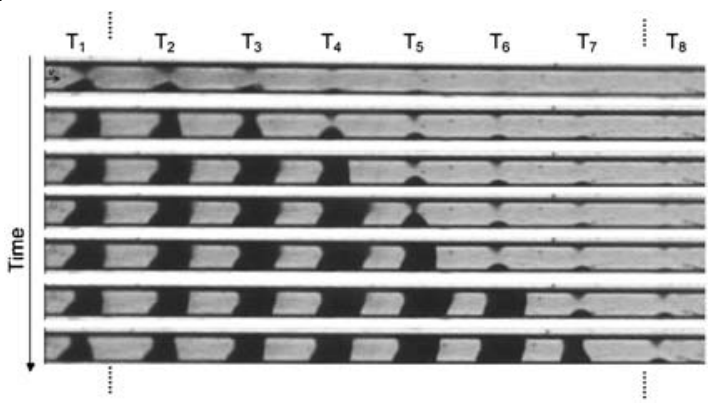

Fig. 5 Fluctuation of magnetic force ( $x$ component) along the microchannel and microscope imaging of the magnetic bead capture. a-b Microchips without, and $\mathbf{c}-\mathbf{d}$ with magnetic tracks are studied.

inter-track gap, each track adds as individual small magnet presenting a negative $\boldsymbol{F}_{\mathbf{m a g}}$ value. Obviously, a compromise between the number of tracks (i.e., the number of plugs and by the way the amount of trapped beads) and the intensity of the repelling magnetic force has to be found. It is the reason why, with the flow velocities applied, an inter-track gap of $200 \mu \mathrm{m}$ appeared as a good compromise for the chip.

In order to extend the present study to other kind of magnetic inks, the effect of the ink properties on the $F_{x}$ gain was evaluated. The dimensionless relative permeability value was varied from 1 to 3,000 and it shows a linear gain increase from 1 to about 20 , for $\mu_{r}=1$ to 10 , respectively. For $\mu_{r}$ values higher than 100 , the gain reaches a plateau. The permeability value of the home-prepared magnetic ink was determined to be around 4 that correspond to a gain of 12 . Working on the optimization of the ink composition, three times higher gain could be reached. In some publications, permalloys are used as magnetic elements [40]. They are often made of nickel (80\%) and iron (20\%), which typically present a relative permeability close to 100 , which would provide a gain of 35 .

The last parameter to be investigated was the length of the track array (data not shown). If the tracks are located outside of the inter-magnet region, the magnetic field lines
The vertical dashed lines on the pictures show the position of the permanent magnets extremities respect to microchannel. $S$ Start, $E$ end of the plug of beads. $L_{\mathrm{P}}$ plug length

will also be attracted to these outer tracks, decreasing the magnetic field and force of the inner tracks. In addition, these tracks will not efficiently trap beads because of their limited magnetic force amplitude.

In the reference design, the channel-track gap is $10 \mu \mathrm{m}$ and inter-track gap is $200 \mu \mathrm{m}$, and the magnetic tracks fit the permanent magnet length ending with 8,17 , and 34 magnetic tracks for 2-, 5-, and 10-mm-long magnet, respectively.

Relative quantification of the magnetic bead capture

As the available binding surface for bioassays is directly correlated to the amount of trapped beads, relative quantification was done comparing a multi-plug vs. a single-plug bead capture (Fig. 7). First, the bead capture was imaged within three microchips with 8,17 , and 34 tracks used with 2-, 5-, and 10-mm-long magnets, respectively. The magnetic bead injection time was increased (the flow velocity was kept constant). From the microscope images, the surface occupied by the beads was extracted. The resulting surfaces obtained from each experimental condition were then normalized by the maximal occupancy value (i.e., value at the plateau) obtained with the 34 -track 
a

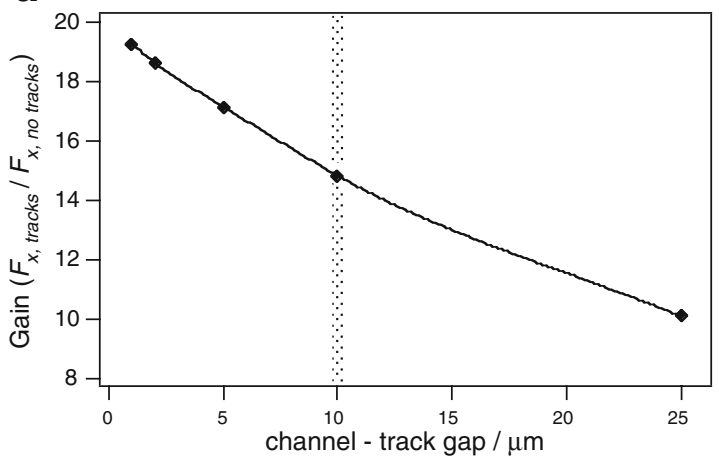

C

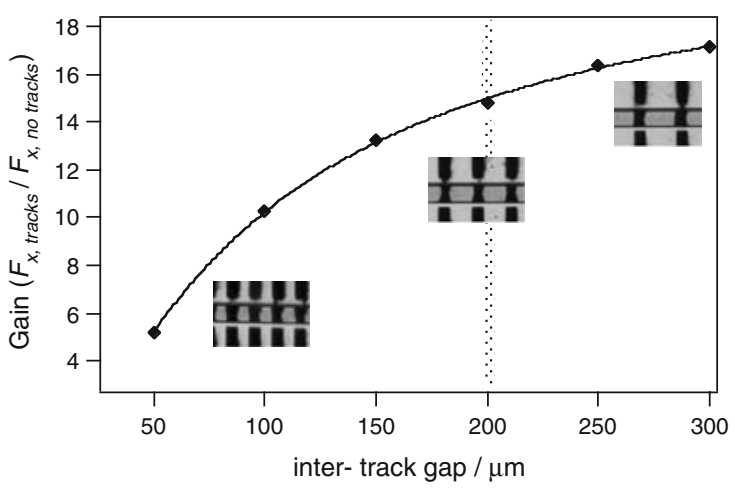

Fig. 6 Influence of the geometrical parameters and magnetic track permeability on the magnetic force. The gain is expressed as the ratio of magnetic forces between the microchip embedding tracks and not. a-b Gain ( $x$ - and $y$ components) in function of the channel-track gap

microchip. When the relative occupancy reaches a plateau (Fig. 7), all the plugs are saturated with beads. As expected, this saturation value increases with the number of tracks.

Afterwards, the same amounts of beads corresponding to the multi-plug saturation were injected in the respective b

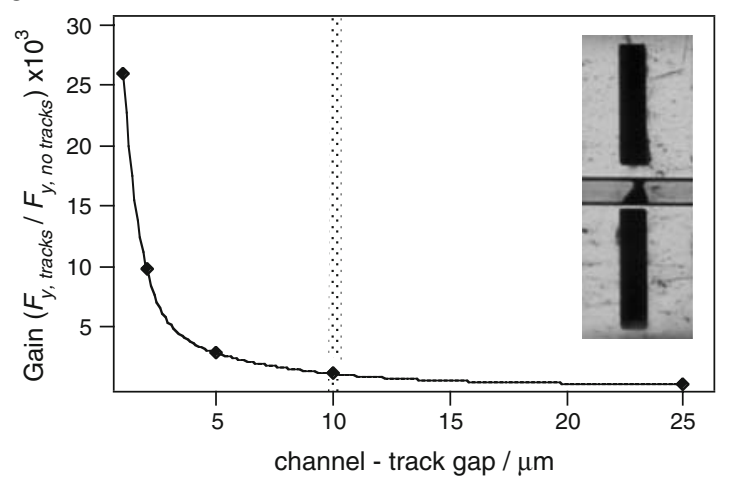

d

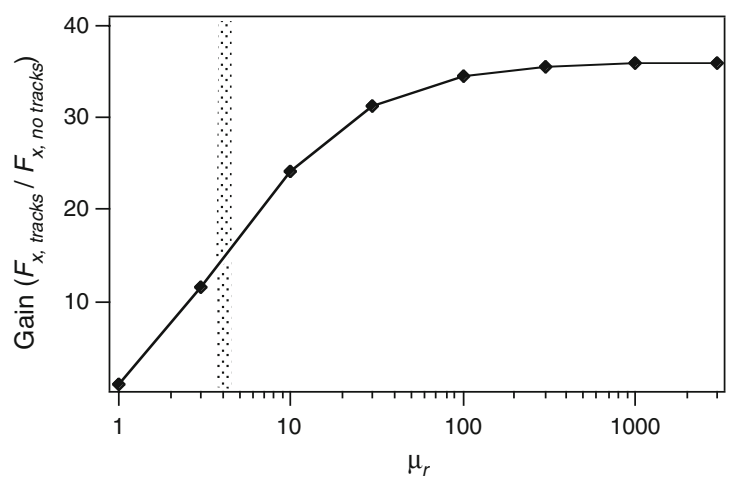

value. $\mathbf{c}-\mathbf{d}$ Gain ( $x$ component) in function of the inter-track gap value. d Generalization of the study to other ink permeability values. The picture tags are microscopic images done in parallel to the simulations. The gray region shows the value of the reference design

microchips without magnetic tracks, at the same flow velocity (horizontal lines with gray markers on Fig. 7). The relative occupancy then represents $10 \%, 29 \%, 31 \%$ of the maximal occupancy for 2-, 5-, and 10-mm-long magnets, respectively, showing a relevant impact of the
Fig. 7 Quantification of the magnetic bead capture with 2-, 5-, and 10-mm-long magnets and comparison with microchip without tracks. The magnetic bead injection time is increased, with the injection protocol set at $250 \mathrm{~nL} / \mathrm{min}$ for injection and $100 \mathrm{~nL} / \mathrm{min}$ for the bead flowing and capture

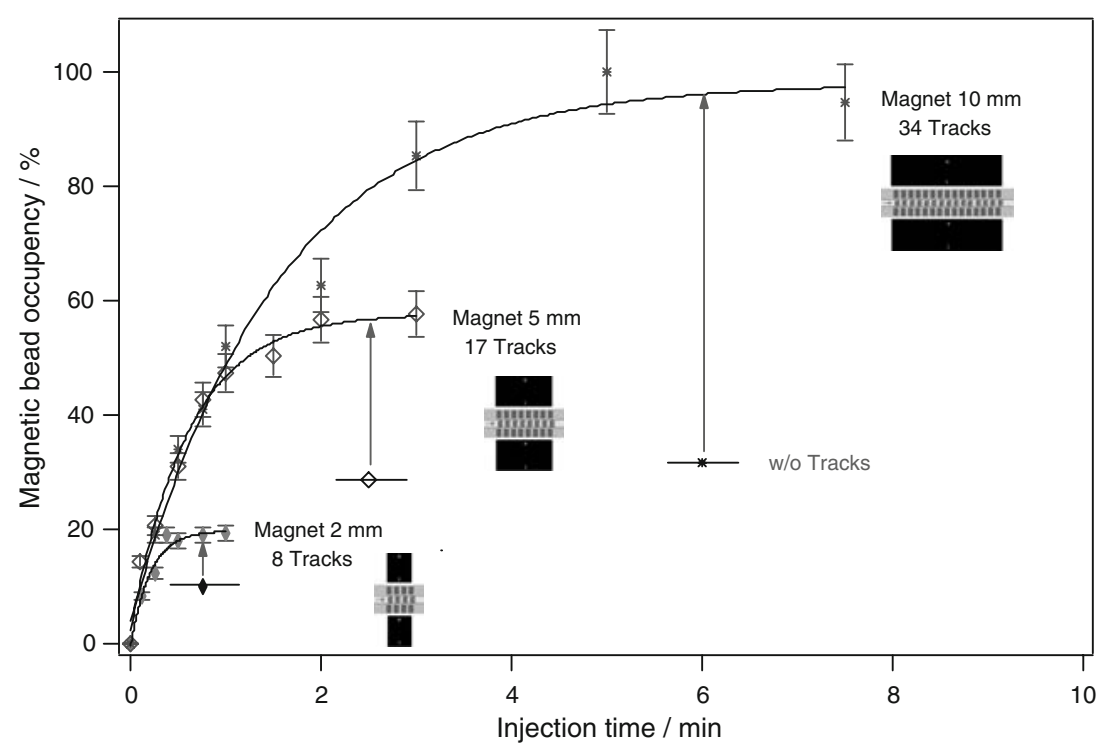


magnetic track incorporation. The difference is actually due to a loss of beads without tracks. For the magnets 5- and 10 -mm-long without tracks, at a given flow rate, approximately $60 \%$ of the beads are lost during the capturing protocol. It is partly due to the magnetic force value, i.e., the magnetic force over drag force ratio, which is lower in the absence of magnetic tracks, as shown in Figs. 5 and 6. For the 2-mm-long magnets, the small magnet length limits the amount of trapped beads as the area where $\boldsymbol{F}_{\text {mag }}$ competes with $\boldsymbol{F}_{\text {drag }}$ has a length which order of magnitude is half of the magnet length (in stopped-flow conditions).

The use of magnetic tracks allows a significant increase in the amount of trapped beads and potentially, the available binding surface for bio-chemical assays. The increase reaches $200 \%$ for the 2- and 5-mm-long magnets and goes up to $300 \%$ for the largest tested. Of course, this value depends on the flow rate and amount of injected beads, as well as the track position (i.e., the number of tracks and consequently the number of plugs).

\section{Conclusions}

This paper introduces a simple way to enhance the magnetic bead capture in a microchannel, and so the available specific binding surface for bio-chemical assays, by locally addressing the magnetic field through magnetic track arrays. Microscope imaging and 2D finite elements simulations were performed considering a microchannel surrounded by two permanent magnets in attraction. Microscope imaging has shown that, for the given flow velocity conditions, the bead capture remains almost constant for a magnet length higher than $5 \mathrm{~mm}$. To enhance the bead capture, $100-\mu \mathrm{m}$-wide magnetic tracks perpendicular to the microchannel axis, were integrated to drive the magnetic field towards specific locations. Therefore, in the gap between each opposite track, a repelling magnetic force retains the beads leading to a multi-plug capture.

The influence of geometrical parameters on the magnetic force, such as the channel-track and inter-track gap values, as well as the magnetic track permeability, were evaluated by numerical simulations, with a microchip operating with 2-mm-long magnets. When the inter-track gap is large, the $x$ component of the force is mainly concerned, leading to an increase of its amplitude $\left(\boldsymbol{F}_{\boldsymbol{x}}\right.$ multiplied by three for a intertrack gap increasing from 50 to $300 \mu \mathrm{m})$. On the other hand, changing the vertical position of the tracks mostly affects the $y$ component of the force $\left(\boldsymbol{F}_{\boldsymbol{y}}\right.$ increased by a factor 25 for a channel-track gap decreasing from 25 to $1 \mu \mathrm{m})$. The closer to the microchannel the tracks are, the larger is the $y$ component force magnitude.

Thanks to the multi-plug capture, the amount of trapped beads can be increased from $200 \%$ for the 8 - and 17 -track to $300 \%$ for the 34 -track reference designs. The increase of the bead amount considerably enhances the binding surface available for in vitro applications, such as immunoaffinity separation. Moreover, the negative force retaining the beads is amplified by passing through the tracks (ten times higher for the inner tracks) enabling a capture at higher flow rates. Finally, the precise positioning of the tracks can overcome the difficult symmetrical positioning of the chip in between the magnets.

It would be interesting to further investigate the growth of the plugs in order to understand more deeply the dependence of the plug length with the fluidic and magnetic parameters, and in particular with the $\boldsymbol{F}_{\text {mag }}$ over $\boldsymbol{F}_{\text {drag }}$ ratio.

Further developments on the present microchip, will be to integrate on-chip electrochemical detection to perform enzyme-based immunoassays, or to embed carbon electrodes to use it as electrospray emitter in order to follow on-line reactions directly by mass spectrometry.

Acknowledgments Prof. Jean-Philippe Ansermet and Dr. Simon Granville from the Laboratory of the Physics of Nanostructured Materials (EPFL, Switzerland) are thanked for the magnetic ink susceptibility measurements. Prof. Heinrich Hofmann and Carlos Morais from the Powder technology laboratory (EPFL, Switzerland) are thanked for their contribution in the magnetic ink density measurements and fruitful discussions. This work was supported by the Fond National Suisse pour la Recherche Scientifique (FN 200020113413 "Analytical tools for fast phosphoproteome analysis", FNPNR 404740-117324 "Supramolecular phases for protein adsorption", CTI 9215.1 "Microfluidic plateform for fast immunoassays").

\section{References}

1. Peoples MC, Karnes HT (2008) Journal of chromatography B: analytical technologies in the biomedical and life sciences 866:14-25

2. Rossier JS, Girault HH (2001) Lab on a Chip - Minituarization for Chemistry and Biology 1:153-157

3. Lionello A, Josserand J, Jensen H, Girault H (2005) Lab Chip 5:254-260

4. Lionello A, Josserand J, Jensen H, Girault H (2005) Lab Chip 5:1096-1103

5. Chen HX, Busnel JM, Gassner AL, Peltre G, Zhang XX, Girault HH (2008) Electrophoresis 29:3414-3421

6. Chen HX, Busnel JM, Peltre G, Zhang XX, Girault HH (2008) Anal Chem 80:9583-9588

7. Morier P, Vollet C, Michel PE, Reymond F, Rossier JS (2004) Electrophoresis 25:3761-3768

8. Shevkoplyas SS, Siegel AC, Westervelt RM, Prentiss MG, Whitesides GM (2007) Lab on a Chip - Miniaturisation for Chemistry and Biology 7:1294-1302

9. Pankhurst QA, Connolly J, Jones SK, Dobson J (2003) J Phys, D Appl Phys 36

10. Ito A, Shinkai M, Honda H, Kobayashi T (2005) J Biosci Bioeng 100:1-11

11. Whitesides GM, Kazlauskas RJ, Josephson L (1983) Trends Biotech 1:144-148

12. Safarik I, Safarikova M (1999) J Chromatogr, B, Biomed Sci Appl 722:33-53 
13. Safarikova M, Safarik I (1999) J Magn Magn Mater 194:108-112

14. Janasek D, Franzke J, Manz A (2006) Nature 442:374-380

15. Pamme N (2006) Lab on a Chip - Miniaturisation for Chemistry and Biology 6:24-38

16. Verpoorte E (2003) Lab Chip 3

17. Gijs MAM (2004) Microfluidics and Nanofluidics 1:22-40

18. Man KH, Bruno Frazier A (2004) J Appl Physi 96:5797-5802

19. Han KH, Frazier AB (2005) Journal of Microelectromechanical Systems 14:1422-1431

20. Pamme N, Wilhelm C (2006) Lab on a Chip - Miniaturisation for Chemistry and Biology 6:974-980

21. Furlani EP (2007) J Phys, D, Appl Phys 40:1313-1319

22. Jung J, Han KH (2008) Appl Phys Lett 93

23. Qu BY, Wu ZY, Fang F, Bai ZM, Yang DZ, Xu SK (2008) Analytical and Bioanalytical Chemistry 392:1317-1324

24. Korecká L, Jankovicová B, Krenková J, Hernychová L, Slováková M, Le-Nell A, Chmelík J, Foret F, Viovy JL, Bílková Z (2008) J Sep Sci 31:507-515

25. Slovakova M, Minc N, Bilkova Z, Smadja C, Faigle W, Fütterer C, Taverna M, Viovy JL (2005) Lab on a Chip - Miniaturisation for Chemistry and Biology 5:935-942

26. Peyman SA, Iles A, Pamme N (2008) Chem Commun 1220-1222

27. Lacharme F, Vandevyver C, Gijs MAM (2008) Anal Chem 80:2905-2910

28. Sista RS, Eckhardt AE, Srinivasan V, Pollack MG, Palanki S, Pamula VK (2008) Lab on a Chip - Miniaturisation for Chemistry and Biology 8:2188-2196

29. Smistrup K, Kjeldsen BG, Reimers JL, Dufva M, Petersen J, Hansen MF (2005) Lab on a Chip - Miniaturisation for Chemistry and Biology 5:1315-1319

30. Minc N, Fütterer C, Dorfman KD, Bancaud A, Gosse C, Goubault C, Viovy JL (2004) Anal Chem 76:3770-3776

31. Smistrup K, Stone HA (2007) Phys Fluids 19
32. Satarkar NS, Zhang W, Eitel RE, Hilt JZ (2009) Lab on a Chip Miniaturisation for Chemistry and Biology

33. Rida A, Gijs MAM (2004) Anal Chem 76:6239-6246

34. Seong GH, Crooks RM (2002) J Am Chem Soc 124:1336013361

35. Biswal SL, Gast AP (2004) Anal Chem 76:6448-6455

36. Wang Y, Zhe J, Chung BTF, Dutta P (2008) Microfluidics and Nanofluidics 4:375-389

37. Lee SH, Van Noort D, Lee JY, Zhang BT, Park TH (2009) Lab on a Chip - Miniaturisation for Chemistry and Biology 9:479-482

38. Hayes MA, Polson NA, Garcia AA (2001) Langmuir 17:28662871

39. Gassner AL, Abonnenc M, Morandini J, Josserand J, Busnel JM, Girault H (2009) Lab Chip

40. Smistrup K, Lund-Olesen T, Hansen MF, Tang PT (2006) J Appl Physi 99

41. Mirowski E, Moreland J, Russek SE, Donahue MJ (2004) Appl Phys Lett 84:1786-1788

42. Siegel AC, Shevkoplyas SS, Weibel DB, Bruzewicz DA, Martinez AW, Whitesides GM (2006) Angew Chem, Int Ed 45:6877-6882

43. Bäuerle D (1988) Appl Phys, B Photophys Laser Chem 46:261270

44. Bäuerle D, Himmelbauer M, Arenholz E (1997) J Photochem Photobiol, A Chem 106:27-30

45. Deng T, Whitesides GM, Radhakrishnan M, Zabow G, Prentiss M (2001) Appl Phys Lett 78:1775-1777

46. Abonnenc M, Dayon L, Perruche B, Lion N, Girault HH (2008) Anal Chem 80:3372-3378

47. Dayon L, Abonnenc M, Prudent M, Lion N, Girault HH (2006) J Mass Spectrom 41:1484-1490

48. Rohner TC, Rossier JS, Girault HH (2001) Anal Chem 73:53535357

49. Bronzeau S, Pamme N (2008) Anal Chim Acta 609:105-112 